Revue d'histoire de l'Amérique française

REVUE D.HISTOIRE DE L'AMÉRIQUE FRANÇAISE

\title{
LAURIN, Serge, Sainte-Agathe-des-Monts. Un siècle et demi d'histoire (Québec, Les Presses de l'Université Laval, 2002), 336
}

p.

\section{Claire Poitras}

Volume 57, numéro 3, hiver 2004

URI : https://id.erudit.org/iderudit/009602ar

DOI : https://doi.org/10.7202/009602ar

Aller au sommaire du numéro

Éditeur(s)

Institut d'histoire de l'Amérique française

ISSN

0035-2357 (imprimé)

1492-1383 (numérique)

Découvrir la revue

Citer ce compte rendu

Poitras, C. (2004). Compte rendu de [LAURIN, Serge, Sainte-Agathe-des-Monts.

Un siècle et demi d'histoire (Québec, Les Presses de l'Université Laval, 2002), 336

p.] Revue d'histoire de l'Amérique française, 57(3), 439-441.

https://doi.org/10.7202/009602ar d'utilisation que vous pouvez consulter en ligne.

https://apropos.erudit.org/fr/usagers/politique-dutilisation/ 
un allégement du rôle de l'État. Sur ces trois thèmes, il accepte la création de trois comités d'études formés majoritairement de représentants des milieux d'affaires qui proposent un vaste programme de gouvernement. Même si le premier ministre prend une certaine distance à l'égard des recommandations des comités, il n'en reste pas moins qu'elles influencent fortement ses politiques. Il est malheureux que les textes du volume n'insistent pas suffisamment sur les rapports de ces trois comités dont la philosophie continue de nos jours à inspirer le gouvernement libéral.

Le volume représente certainement un bilan précieux pour comprendre à la fois la personnalité de Robert Bourassa et les décisions de son gouvernement. C'est d'autant plus utile que ce premier ministre a dirigé les destinées du Québec pendant une longue période. On doit en remercier les organisateurs du colloque qui ont fait œuvre utile notamment en amenant ses proches en politique à ramasser leurs souvenirs, ce qu'ils n'auraient jamais fait si on ne les avait sollicités. Même s'ils sont élogieux, leurs témoignages seront précieux pour les historiens de demain qui se pencheront sur cette période mouvementée de l'histoire du Québec. L’ensemble des textes du volume ne représente néanmoins qu'une première ébauche du personnage qui ne saurait remplacer un travail en profondeur à partir des archives.

JACQUES ROUILLARD

Département d'histoire

Université de Montréal

LAURIN, Serge, Sainte-Agathe-des-Monts. Un siècle et demi d'histoire (Québec, Les Presses de I'Université Laval, 2002), 336 p.

Réalisée par l'auteur de l'histoire régionale des Laurentides publiées aux Presses de l'IQRC en 1989, cette monographie d'histoire locale retrace les principales transformations d'une petite ville laurentienne depuis sa fondation en 1849 jusqu'à nos jours. Il s'agit d'un ouvrage commandé par le Comité du patrimoine de Sainte-Agathe-des-Monts dans le cadre du $150^{\mathrm{e}}$ anniversaire de la fondation de la municipalité. La directive du commanditaire était claire : la monographie historique doit s'inscrire «en rupture avec les ouvrages généralement produits dans ces cas, à savoir la forme d'album qui se résume à la rédaction de quelques textes anecdotiques entrelardés de photos d'époque et, surtout, mettant l'accent sur la liste des curés, des premiers colons ou celle de quelques personnages célèbres par leurs exploits ou leur originalité» (p. 1). L'auteur a su relever 
ce défi, tout en n'abandonnant pas totalement les caractéristiques propres à la monographie d'histoire locale. Ainsi, Laurin met au jour le rôle des élites locales et des différents acteurs qui ont façonné le paysage urbain agathois. En outre, compte tenu du statut de lieu de villégiature de cette petite ville, l'apport des touristes à son développement n'est pas négligeable. L'auteur expose les forces internes et externes à l'œuvre et les conflits que leur rencontre sont à même d'alimenter.

Divisé en cinq parties comprenant au total dix chapitres et une conclusion générale, l'ouvrage nous conduit à travers les différentes étapes du développement de Sainte-Agathe-des-Monts, depuis son établissement dans les années 1840 comme terre d'accueil des colons venus défricher la forêt jusqu'à ses transformations récentes en tant que ville de services en quête d'identité. Bien qu'elle soit située dans une région administrative - les Laurentides qui connaît depuis les années 1990 un important essor démographique en raison de l'étalement urbain du Grand Montréal, Saint-Agathe n'est pas parvenue à profiter des retombées de cette croissance comme d'autres localités laurentiennes (par exemple Saint-Sauveur ou Mont-Tremblant) ont su le faire. Comment expliquer ces différences infrarégionales? L'histoire vient à la rescousse pour nous permettre de faire ressortir les contrastes relatifs au développement urbain prévalant au sein d'une même région.

À la fin $\mathrm{du} \mathrm{xIX}^{\mathrm{e}}$ siècle, l'urbanisation conduit au démembrement de la paroisse de Sainte-Agathe et à la constitution de la ville de Saint-Agathedes-Monts. Cette division territoriale découle de divergences de points de vue quant aux activités à privilégier : les urbains veulent du développement économique, tandis que les ruraux souhaitent préserver la vocation agricole du milieu. Pour autant, une fois constituée en ville, Saint-Agathedes-Monts n'est pas immunisée contre les conflits internes quant aux orientations économiques à privilégier.

La périodisation proposée par Laurin s'appuie sur des changements socio-économiques ayant des incidences sur les modes d'occupation du territoire et sur l'organisation sociale : les débuts avec l'arrivée des colons, les retombées économiques de la mise en service du chemin de fer en 1892, l'apogée de la ville en tant que capitale touristique des Laurentides entre 1915 et 1947, les tentatives de repositionnement stratégique du statut de pôle touristique en raison de la compétition accrue que se livrent les villes de la région entre 1947 et 1976, l'échec de la stratégie d'industrialisation et la tertiarisation de l'économie entre 1976 et 2002. Le portrait général qui se dégage de l'analyse de ces mutations socio-économiques est celui d'une ville de projets avortés ou ratés. Cela est en grande partie 
dû aux stratégies de développement misant sur un nombre très limité de secteurs d'activité. Au cours du $\mathrm{xx}^{\mathrm{e}}$ siècle, c'est surtout le tourisme et la lutte antituberculeuse - entre 1898 et 1912 plusieurs sanatoriums sont aménagés - qui ont fait la notoriété de Saint-Agathe-des-Monts, et ce, à l'échelle provinciale. À partir du moment où ces secteurs d'activité ont décliné, toute l'économie locale s'est trouvée fragilisée. À maintes reprises, la population locale a dû s'accommoder de telles circonstances. L'histoire de la petite ville est ainsi caractérisée par plusieurs tentatives de relance dont la très grande majorité s'est révélée infructueuse.

L’ouvrage est illustré de photographies anciennes et récentes dont l'éventail permet de maintenir un équilibre entre, d'une part, les individus et les groupes sociaux qui ont contribué au développement de la ville et, d'autre part, le paysage urbain modelé par ces acteurs. Par ailleurs, en ce qui concerne la cartographie, nous ne disposons pas des représentations nécessaires pour bien saisir les dynamiques territoriales à l'œuvre. À plusieurs moments, il est clair que certains processus comme le déploiement des nouveaux modes de transport transforment l'organisation spatiale de la région. Comment Saint-Agathe-des-Monts s'inscrit-elle dans la nouvelle hiérarchie créée par les réseaux de transport? Autrement dit, il aurait été utile de joindre des cartes localisant la ville étudiée dans sa région. Il s'agit là d'une des difficultés auxquelles se heurtent fréquemment les monographies d'histoire locale, à savoir comment faire ressortir la spécificité d'un milieu donné tout en tenant compte d'un contexte géographique plus large. Cela dit, le livre de Laurin renouvelle le genre, notamment par ses analyses détaillées qui mettent en lumière les effets locaux des transformations socio-économiques à même d'influencer le devenir d'une petite ville : la mise en place d'une économie de subsistance, le développement de l'industrie touristique, la venue de nouvelles populations, l'arrivée de la grande industrie, la modernisation des institutions publiques, y inclus les municipalités, et, plus récemment, la mondialisation de l'économie. L'idée la plus originale développée par l'auteur — qu'il avait déjà proposée dans son ouvrage sur l'histoire des Laurentides - est celle du phénomène de la villégiaturbanisation selon lequel les résidences secondaires sont transformées en résidences permanentes modifiant, du coup, la composition démographique d'un milieu. Contrairement à plusieurs villes des Basses-Laurentides, Sainte-Agathe-des-Monts n’a pas encore été touchée par ce phénomène mais ce n'est sans doute qu'une question de temps. 